
\title{
Enumeration, Counting, and Random Generation of Ladder
}

\section{Lotteries*}

\author{
Katsuhisa YAMANAKA $^{\dagger \mathrm{a})}$ and Shin-ichi NAKANO ${ }^{\dagger \dagger \mathrm{b})}$, Members
}

\begin{abstract}
SUMMARY A ladder lottery, known as "Amidakuji" in Japan, is one of the most popular lotteries. In this paper, we consider the problems of enumeration, counting, and random generation of the ladder lotteries. For given two positive integers $n$ and $b$, we give algorithms of enumeration, counting, and random generation of ladder lotteries with $n$ lines and $b$ bars. The running time of the enumeration algorithm is $\mathrm{O}(n+b)$ time for each. The running time of the counting algorithm is $\mathrm{O}\left(n b^{3}\right)$ time. The random generation algorithm takes $\mathrm{O}\left(n b^{3}\right)$ time for preprocess, and then it generates a ladder lottery in $\mathrm{O}(n+b)$ for each uniformly at random.

key words: enumeration, counting, random generation, ladder lottery
\end{abstract}

\section{Introduction}

A ladder lottery, known as "Amidakuji" in Japan, is one of the most popular lotteries for kids. It is often used to assign roles to members in a group. Imagine that a group of four members $A, B, C$, and $D$ wish to determine their group leader using a ladder lottery. First, four vertical lines are drawn, then each member chooses a vertical line. See Fig. 1 (a). Next, a check mark (which represents an assignment of the leader) and some horizontal lines are drawn, as shown in Fig. 1 (b). The derived one is called a ladder lottery, and it represents an assignment. In this example the leader is assigned to $D$ since the top-to-bottom route from $D$ ends at the check mark. (We will explain the route soon.) In Fig. 1 (b), the route is drawn as a dotted line.

Formally, a ladder lottery is a network with $n \geq 2$ vertical lines (lines for short) and $b$ horizontal lines (bars for short) each of which connects two consecutive vertical lines. We count the lines from left to right and call the $i$-th line from the left $i$-th line. See Fig. 2 for an example. The top ends of the lines correspond to a permutation $\pi=\left(p_{1}, p_{2}, \ldots, p_{n}\right)$ of $[n]=\{1,2, \ldots, n\}$, and the bottom ends of the lines correspond to the identity permutation $\iota=(1,2, \ldots, n)$ and they satisfy the following rule. Each $p_{i}$ in $\pi$ starts the top end of the $i$-th line, then goes down along the line; whenever $p_{i}$ meets an end of a bar, $p_{i}$ goes horizontally along the bar to the other end, and then goes down

Manuscript received April 6, 2016.

Manuscript revised July 29, 2016.

Manuscript publicized December 21, 2016.

${ }^{\dagger}$ The author is with the Faculty of Science and Engineering, Iwate University, Morioka-shi, 020-8551 Japan.

${ }^{\dagger \dagger}$ The author is with the School of Science and Technology, Gunma University, Kiryu-shi, 376-8515 Japan.

${ }^{*}$ A preliminary version of this paper was presented at the 9 th International Frontiers of Algorithmics Workshop.

a)E-mail: yamanaka@cis.iwate-u.ac.jp

b) E-mail: nakano@cs.gunma-u.ac.jp

DOI: $10.1587 /$ transinf.2016FCP0015 again. Finally, $p_{i}$ must reach the bottom end of the $p_{i}$-th line. Each bar corresponds to a modification of the current permutation by swapping the two neighboring elements.

A ladder lottery appears in a variety of areas. First, a ladder lottery of the reverse permutation $(n, n-1, \ldots, 1)$ corresponds to a pseudoline arrangement in discrete geometry [9]. By replacing bars as intersections of pseudolines, ladder lotteries can be regarded as pseudoline arrangements, and it is observed that there is a one-to-one correspondence between pseudoline arrangements and "optimal" ladder lotteries of a reverse permutation [9]. A ladder lottery of a permutation is optimal if it has the minimum number of bars among ladder lotteries of the permutation. Second, it is strongly related to primitive sorting networks, which are deeply investigated by Knuth [4]. Third, in algebraic combinatorics, a reduced decomposition (by adjacent transpositions) of a permutation corresponds to a ladder lottery of the permutation with the minimum number of bars [5].

In this paper we consider the problems of enumeration, counting, and random generation of ladder lotteries with $n$ lines and $b$ bars. As mentioned above, ladder lotteries have relations with many other discrete objects, and hence they are interesting objects to be investigated. For optimal ladder lotteries, there are some results on counting and enumeration. Yamanaka et al. [9] proposed an algorithm that enumerates all optimal ladder lotteries of a given permutation in $\mathrm{O}(1)$ time for each. Besides, they counted the number of ladder lotteries of a reverse permutation for $n=11$ using the enumeration algorithm. Samuel [7] improved the result and reported the number for $n=12$. Kawahara et al. [3] improved the results again. They counted the number of such ladder lotteries for $n=13$ using $\pi \mathrm{DD}$, which is a variation of decision diagrams. The current best record is the number for $n=15$ by Tanaka [7]. See [7] for further details. However, it seems to be difficult to efficiently count the numbers for large $n$. This motivates us to deal with ladder lotteries with $n$ lines and $b$ bars. For these ladder lotteries, a simple and natural binary code is known [1] (the code will be introduced in the next section). Using the code, we propose an efficient counting algorithm of ladder lotteries with $n$ lines and $b$ bars. The running time of the algorithm is $\mathrm{O}\left(n b^{3}\right)$ time. As a by-product, we obtain a random-generation algorithm which generates a ladder lottery in $\mathrm{O}(n+b)$ time for each uniformly at random. Besides, we proposed a simple enumeration algorithm which generates a ladder lottery in $\mathrm{O}(n+b)$ time for each. 


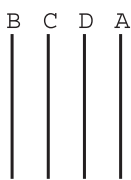

(a)

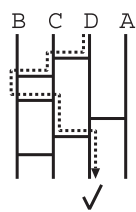

(b)
Fig. 1 An example of a ladder lottery.

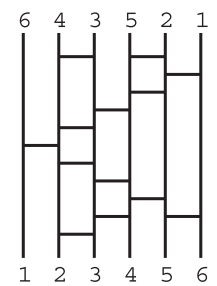

Fig. 2 A ladder lottery of $(6,4,3,5,2,1)$.

\section{Preliminaries}

We assume that a ladder lottery is given as a graph structure in which the vertices are the set of the top and bottom endpoints of lines and endpoints of bars, and edges are line segments between vertices. Suppose that, for an endpoint $v$ and its adjacent one $u$, we can recognize the direction (top, bottom, left, and right) of $u$ from $v$.

\subsection{Code of Ladder Lotteries}

In this subsection, we review a code of ladder lotteries in [1]. Using this code, we will design three algorithms in the following sections.

Let $L$ be a ladder lottery with $n$ lines and $b$ bars. We first divide each bar of $L$ into two horizontal line-segments, called half-bars. The left half of a bar is called an l-bar (left half-bar) and the right half of a bar is called an $r$-bar (right half-bar). We regard each original bar as a pair of an 1-bar and an r-bar. Thus $L$ has $2 b$ half-bars. The division results in $n$ connected components, each of which consists of one line and some half-bars attached to the line.

We can encode how half-bars are attached to the $i$-th line, as follows. Let $\left\langle b_{1}, b_{2}, \ldots\right\rangle$ be the sequence of halfbars attached to the $i$-th line appearing from top to bottom. We replace $b_{i}$ with $\theta$ if $b_{i}$ is an r-bar, and with 1 if $b_{i}$ is an l-bar. Then appending a $\theta$ to indicate the end-of-line. This results in the code of the $i$-th line, which is denoted by $C(i)$. Concatenating those codes $C(1), C(2), \ldots, C(n)$ results in the code $C(L)$ for $L$. For example, for the ladder lottery in Fig. $2 C(1)=10, C(2)=110110, C(3)=01001100$, $C(4)=1100100, C(5)=010010, C(6)=000$, and

$$
C(L)=10110110010011001100100010010000 .
$$

Since the code contains two bits for each bar and one bit for each end-of-line, its length is $n+2 b$ bits.

\subsection{Reconstruction from the Code}

Now we explain how to reconstruct the original ladder lottery from the code.

In the code, a $\otimes$ represents either an r-bar or an endof-line. Hence, we need to recognize the end-of-lines to partite $C(L)$ into $C(1), C(2), \ldots, C(n)$. After then, it is easy to reconstruct original bars by connecting the corresponding l-bars and r-bars, since the $k$-th l-bar of the $i$-th line and the $k$-th r-bar of the $(i+1)$-th line correspond to an original bar. Figure 3 shows an example of the reconstruction of the ladder lottery in Fig. 2 from its code.

We now explain how to recognize the end-of-lines. Since the first line has only l-bars, the first consecutive $1 \mathrm{~s}$ correspond to the 1-bars of the first line, so the first $\theta$ is the end-of-line of the first line. Now we assume that the end-ofline for the $(i-1)$-th line is recognized and we are now going to recognize the end-of-line for the $i$-th line. We know the number, say $k$, of 1-bars attached to the $(i-1)$-th line, and it equals to the number of r-bars attached to the $i$-th line. Then the end-of-line for the $i$-th line is the $(k+1)$-th $\theta$ after the end-of-line for the $(i-1)$-th line.

Theorem 1 ([1]): Let $L$ be a ladder lottery with $n$ lines and $b$ bars. One can encode $L$ into a bitstring of length $n+2 b$. Both encoding and decoding can be done in $\mathrm{O}(n+b)$ time.

\subsection{Pre-Ladder and Its Code}

Let $L$ be a ladder lottery with $n$ lines and $b$ bars, and let $C(L)$ be the code of $L$. We define a substructure of $L$, as follows. Let $P(C(L))$ be the bitstring derived from $C(L)$ by removing the second last bit, and $P(L)$ be the substructure of $L$ derived by "decoding" $P(C(L))$. Intuitively, $P(L)$ is the substructure of $L$ only missing either a half-bar or an end-of-line, corresponding to the second last bit. Similarly $P(P(C(L)))$ is the bitstring derived from $P(C(L))$ by removing the second last bit, and $P(P(L))$ be the corresponding substructure of $L$. Similarly, we define $P(P(P(C(L)))), P(P(P(P(C(L))))), \ldots$. We assume that a pre-ladder has at least two lines. See Fig. 4 for an example. In the figure, end-of-lines are depicted as black circles except for the end-of-line of the rightmost line, which is depicted as a white circle. We say each of those substructures (including $L$ itself) a pre-ladder of $L$, and the sequence $L, P(L), P(P(L)), \ldots$ the removing sequence of $L$. Note that each ladder lottery has the unique removing sequence. From the assumption, the last pre-ladder of the sequence has exactly two lines and the rightmost (second) line has no half bars. A pre-ladder possibly has unmatched l-bars only at the two rightmost lines.

\section{Enumeration}

Let $\mathcal{S}_{n, b}$ be the set of ladder lotteries with $n$ lines and $b$ bars. In this section, we consider the problem of enumerating all ladder lotteries in $\mathcal{S}_{n, b}$. We have presented an algorithm that 


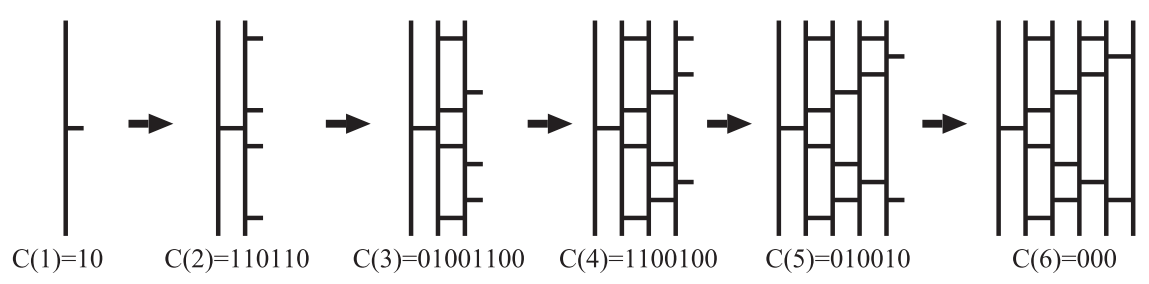

Fig. 3 An example of the reconstruction from the code.

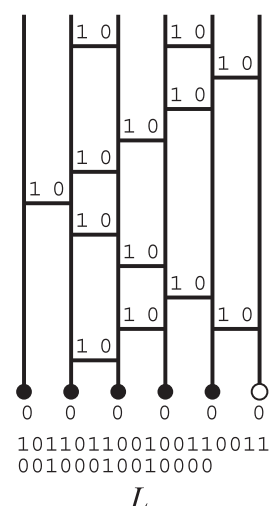

$L$

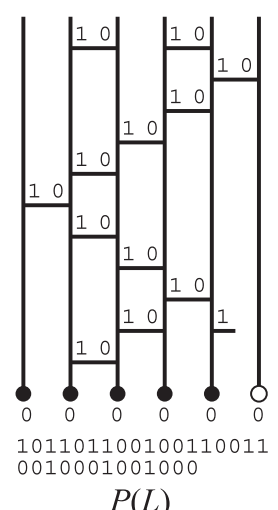

$P(L)$

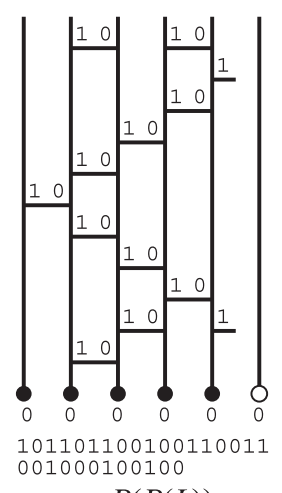

$P(P(L))$

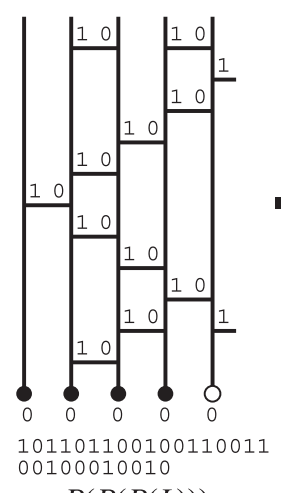

$P(P(P(L)))$

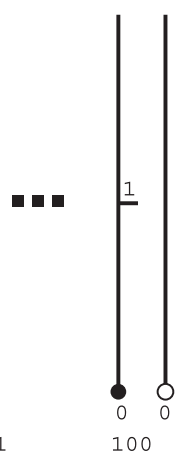

$P(P(\ldots P(L) \ldots))$

Fig. 4 Pre-ladders derived from $L$.

enumerates all "optimal" ladder lotteries of a given permutation [9]. However, this algorithm cannot applied to the problem, since $\mathcal{S}_{n, b}$ includes (non-optimal) ladder lotteries. In this section, we propose a simple enumeration algorithm for $\mathcal{S}_{n, b}$.

Our enumeration algorithm is based on reverse search [2]. We first define a forest structure in which each leaf one-to-one corresponds to some ladder lottery in $\mathcal{S}_{n, b}$. Then, by traversing the forest, we can enumerate all leaves of the forest, and all corresponding ladder lotteries in $\mathcal{S}_{n, b}$. We designed several enumeration algorithms based on similar (but distinct) tree structures [6], [8], [9].

Let $L$ be a ladder lottery in $\mathcal{S}_{n, b}$. By merging the removing sequence for every $L \in \mathcal{S}_{n, b}$, we have the forest, called family forest $F_{n, b}$. Recall that each ladder lottery has the unique removing sequence and each removing sequence ends up with a pre-ladder with exactly two lines and no halfbar attached to the second line. Hence, each root of the family forest is such a pre-ladder and leaves of the family forest $F_{n, b}$ one-to-one corresponds to ladder lottery in $\mathcal{S}_{n, b}$. See Fig. 5 for an example.

By traversing $F_{n, b}$, we can enumerate all the ladder lotteries corresponding to the leaves. We have the following theorem. The proof is given in Appendix.

Theorem 2: One can enumerate all ladder lotteries with $n$ lines and $b$ bars in $\mathrm{O}(n+b)$ time for each. Our algorithm uses $\mathrm{O}(n+b)$ space.

\section{Counting}

In this section we consider a counting problem. Given two positive integers $n \geq 2$ and $b \geq 0$, we wish to count the number of ladder lotteries with $n$ lines and $b$ bars. Using the enumeration algorithm in the previous section, we can count such ladder lotteries one by one, but very slowly. This method takes $\Omega\left(\left|\mathcal{S}_{n, b}\right|\right)$ time, which may be exponential on $n$ and $b$. In this section, we propose an efficient counting algorithm. Our algorithm does not count ladder lotteries one by one, but counts each "type" of pre-ladders all together, and runs in polynomial time ${ }^{\dagger}$.

We now define the type for each pre-ladder. A preladder $R$ is type $t(\ell, h, p, q)$ if $R$ satisfies the following conditions:

(a) $R$ contains $\ell \geq 2$ lines;

(b) $R$ contains $h \geq p+q$ half-bars (Each bar is counted as two half-bars);

(c) $p$ unmatched 1-bars are attached to the $(\ell-1)$-th line; and

(d) $q$ unmatched l-bars are attached to the $\ell$-th line.

For example, the pre-ladder $P(L)$ in Fig. 4 is type $t(6,25,1,0)$. Note that a pre-ladder is a ladder lottery with $n$ lines and $b$ bars if and only if it is of type $t(n, 2 b, 0,0)$. We denote by $T(\ell, h, p, q)$ the set of pre-ladders of type $t(\ell, h, p, q)$. We give a useful recurrence for $|T(\ell, h, p, q)|$.

We have the following four cases.

Case 1: $h<p+q$ or $\ell<2$.

$|T(\ell, h, p, q)|=0$ holds, since $h \geq p+q$ and $\ell \geq 2$ hold for any pre-ladder.

Case 2: $\ell=2, q=0$, and $h=p$ Clearly such pre-ladder is unique, so $|T(\ell, h, p, q)|=1$ holds.

\footnotetext{
${ }^{\dagger}$ We assume that $n$ and $b$ are coded in unary codes.
} 


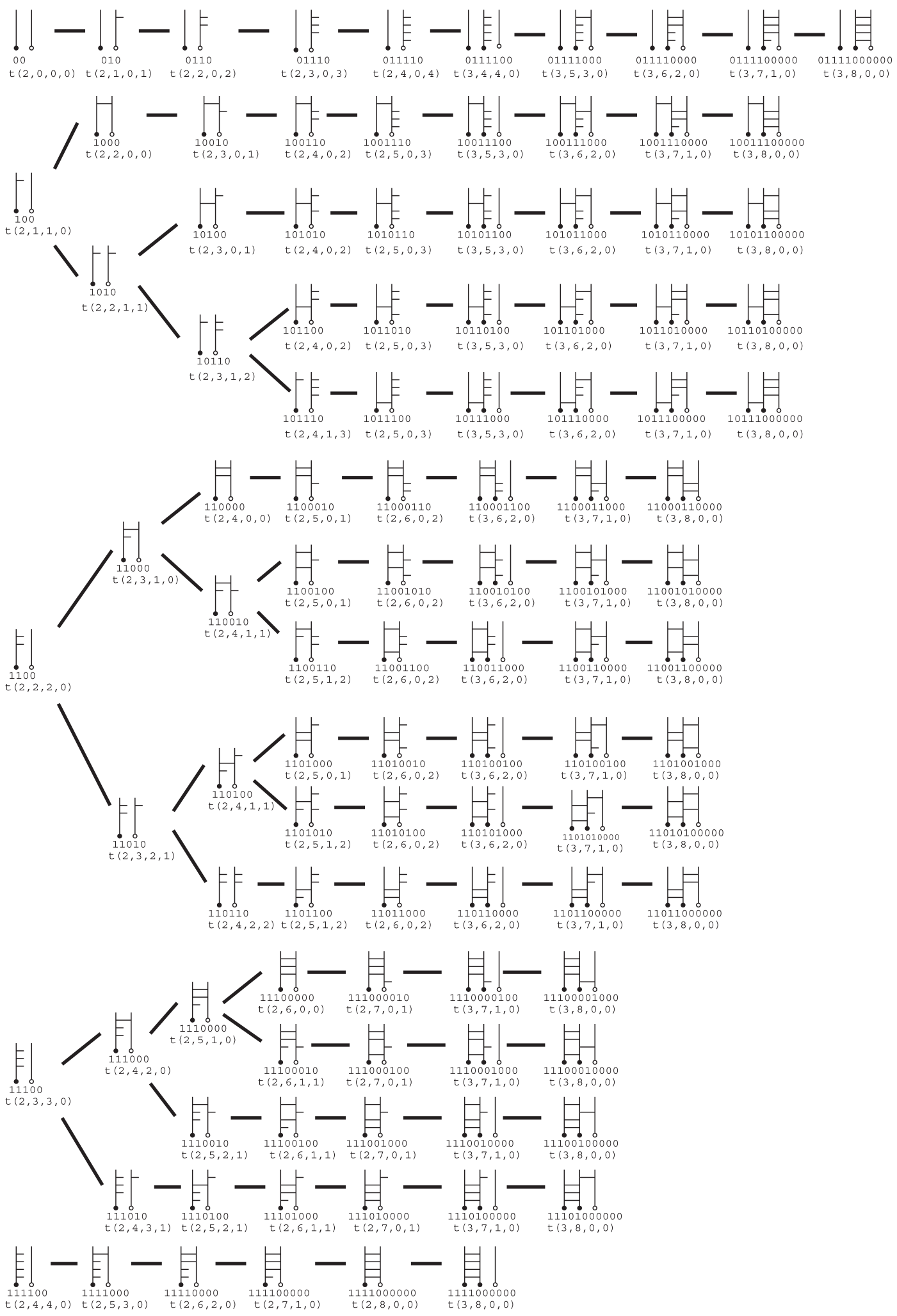

Fig. 5 The family forest $F_{3,4}$.

Case 3: $(\ell \geq 3$ or $h>p)$ and $q=0$

Let $R$ be a pre-ladder of type $t(\ell, h, p, q)$. The second last bit of $C(R)$ is always 0 . (Otherwise, the $\ell$-th line has an l-bar, a contradiction.) The second last bit $\theta$ in $C(R)$ repre- sents either an r-bar of $\ell$-th line or the end-of-line of $(\ell-1)$-th line. For the former case $P(R)$ is type $t(\ell, h-1, p+1,0)$. For the latter case $P(R)$ is type $t(\ell-1, h, 0, p)$. For any distinct $R_{1}$ and $R_{2}$ of $t(\ell, h, p, q)$ with $(\ell \geq 3$ or $h>p)$ and $q=0, P\left(R_{1}\right)$ 


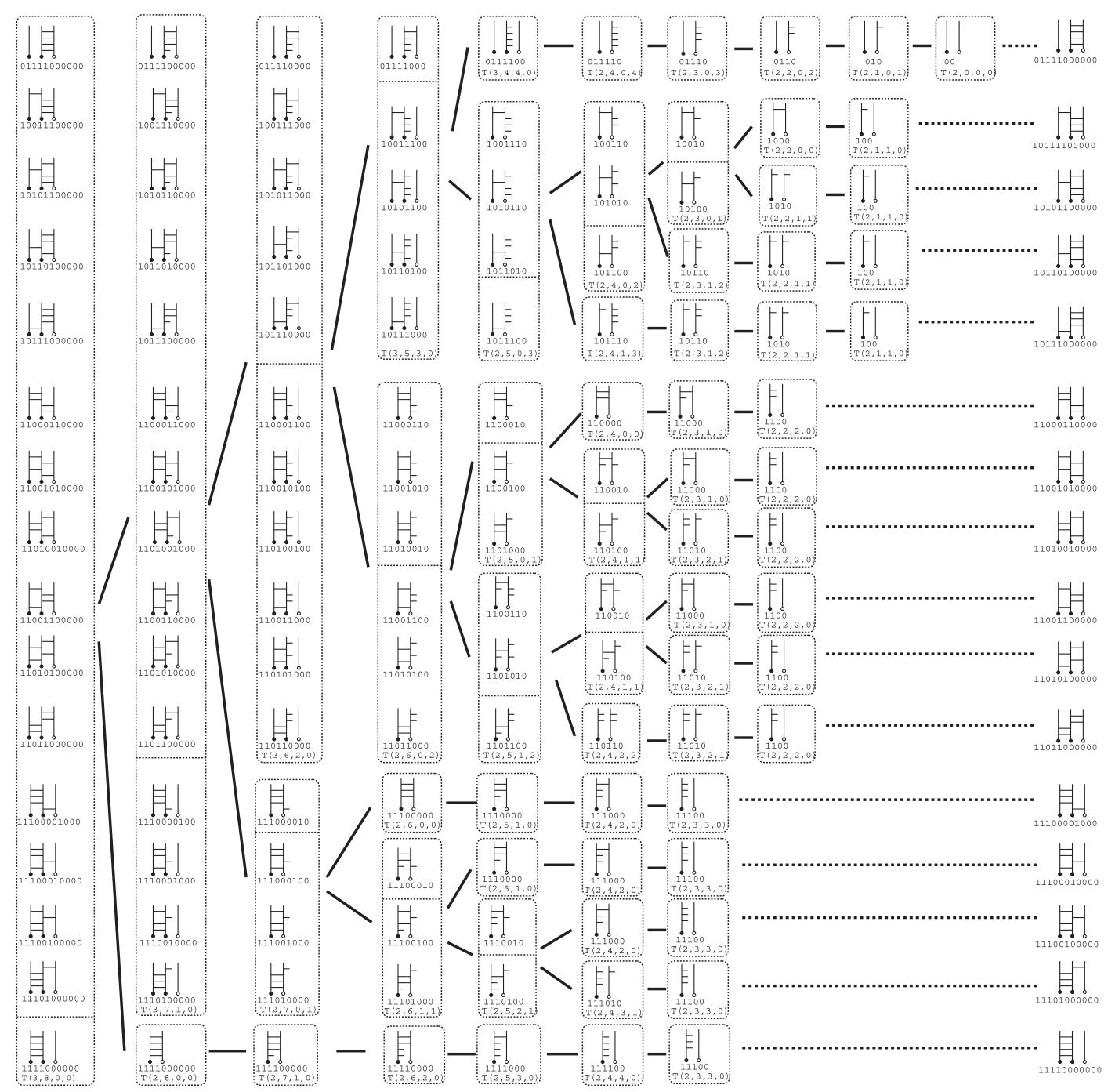

Fig. 6 The recurrence for $|T(3,8,0,0)|$.

and $P\left(R_{2}\right)$ are distinct. Hence, we have an injection from $T(\ell, h, p, q)$ to $T(\ell, h-1, p+1,0) \cup T(\ell-1, h, 0, p)$. Now we also have a surjection, as follows. Let $R^{\prime}$ be a pre-ladder of type $t(\ell, h-1, p+1,0)$, and let $D$ be the code obtained from $C\left(R^{\prime}\right)$ by appending $\Theta$ as the second last bit. Then, the appended $\theta$ represents an $\mathrm{r}$-bar of $\ell$-th line which matches with an unmatched 1-bar of $(\ell-1)$-th line. Hence, the preladder corresponding to $D$ is of type $t(\ell, h, p, 0)$. The similar discussion works for a pre-ladder of type $t(\ell-1, h, 0, p)$. Thus $|T(\ell, h, p, 0)|=|T(\ell, h-1, p+1,0)|+|T(\ell-1, h, 0, p)|$ holds.

Case 4: $h \geq p+q$ and $q>0$.

Let $R$ be a pre-ladder of type $t(\ell, h, p, q)$. The second last bit in $C(R)$ is either $\theta$ or 1 . If the second last bit of $C(R)$ is $\theta$, then it represents an r-bar attached to $\ell$-th line. Thus, $P(R)$ is type $t(\ell, h-1, p+1, q)$. Otherwise, the second last bit of $C(R)$ is 1 , then it represents an 1-bar attached to $\ell$-th line. Hence, $P(R)$ is type $t(\ell, h-1, p, q-1)$. Note that, for any distinct $R_{1}$ and $R_{2}$ of $t(\ell, h, p, q)$ with $h \geq p+q$ and $q>0$,
$P\left(R_{1}\right)$ and $P\left(R_{2}\right)$ are distinct. Let $R^{\prime}$ be a pre-ladder of type $t(\ell, h-1, p+1, q)$, and let $D_{0}$ be the code obtained from $C\left(R^{\prime}\right)$ by appending $\theta$ as the second last bit. Then the appended $\theta$ represents an r-bar which matches with an unmatched l-bar of $(\ell-1)$-th line. Hence the pre-ladder corresponding $D_{0}$ is of type $t(\ell, h, p, 0)$. Let $R^{\prime \prime}$ be a pre-ladder of type $t(\ell, h-$ $1, p, q-1)$, and let $D_{1}$ be the code obtained from $C\left(R^{\prime \prime}\right)$ by appending 1 as the second last bit. Then the appended 1 represents an unmatched l-bar of $\ell$-th line. Hence the preladder corresponding $D_{1}$ is also of type $t(\ell, h, p, 0)$. Thus $|T(\ell, h, p, q)|=|T(\ell, h-1, p+1, q)|+|T(\ell, h-1, p, q-1)|$ holds.

For example, Fig. 6 shows the recurrence for $|T(3,8,0,0)|$. By the recurrence, we have the following lemma.

Lemma 1: For four non-negative integers $\ell, h, p$, and $q$, 


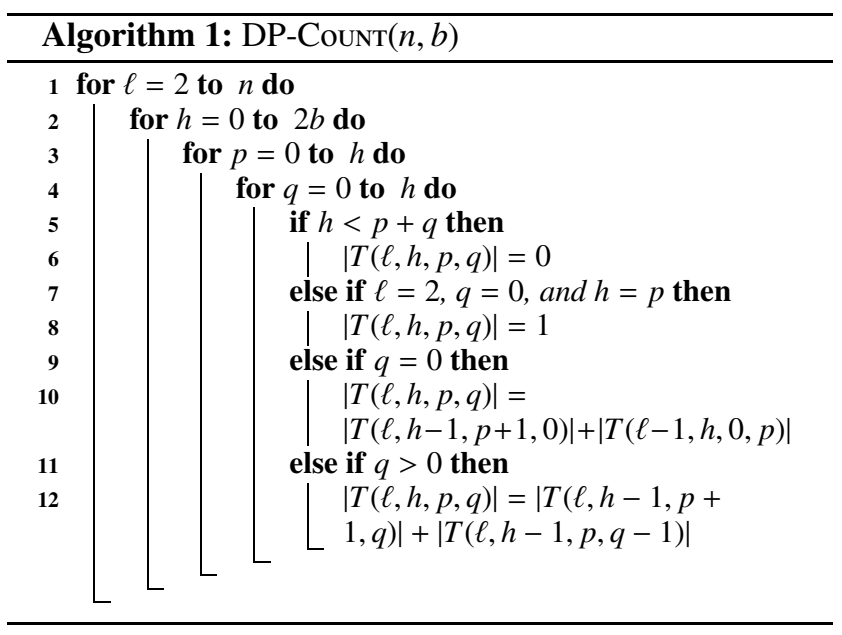

$$
\begin{aligned}
& |T(\ell, h, p, q)| \\
& \quad=\left\{\begin{array}{l}
0 \quad \text { if } h<p+q \text { or } \ell<2 \\
1 \quad \text { if } \ell=2, q=0, \text { and } h=p \\
|T(\ell, h-1, p+1,0)|+|T(\ell-1, h, 0, p)| \\
\text { if }(\ell \geq 3 \text { or } h>p) \text { and } q=0 \\
|T(\ell, h-1, p+1, q)|+|T(\ell, h-1, p, q-1)| \\
\text { if } h \geq p+q \text { and } q>0
\end{array}\right.
\end{aligned}
$$

Based on the recurrence above, Algorithm 1 computes the number of ladder lotteries with $n$ lines and $b$ bars. Algorithm 1 is a dynamic programming algorithm on the table of types. The number of entries is $n b^{3}$, and each entry is calculated in constant time, so the total running time is $\mathrm{O}\left(n b^{3}\right)$. As a byproduct the number of ladder lotteries with $n^{\prime} \leq n$ lines and $b^{\prime} \leq b$ bars are also computed.

Theorem 3: The number of ladder lotteries with $n^{\prime} \leq n$ lines and $b^{\prime} \leq b$ bars can be calculated in $\mathrm{O}\left(n b^{3}\right)$ time in total.

\section{Random Generation}

In this section we consider random generations of ladder lotteries. The recurrence in Lemma 1 generates a tree structure among the types (see an example in Fig. 6), in which each path from the root to a leaf one-to-one corresponds to some ladder lottery of type $t(n, 2 b, 0,0)$. The choice of $i$-th generation type decides the meaning of the $(i+1)$-th last bit of the code. (Here the root belongs to the first generation.)

The table generated by Algorithm 1 tells us the number of leaves in the subtree rooted at each type. We can choose a random path from the root to some leaf, by repeatedly choosing some child of the current type so that each leaf has an equal chance to be reached. Thus we can generate ladder lotteries, uniformly at random.

Our algorithm is shown in Algorithm 2. Suppose that we are now at a type $T(\ell, h, p, q)$ in the tree structure, and $T\left(\ell_{1}, h_{1}, p_{1}, q_{1}\right)$ and $T\left(\ell_{2}, h_{2}, p_{2}, q_{2}\right)$ are the two child types of $T(\ell, h, p, q)$. Algorithm 2 computes a random integer,

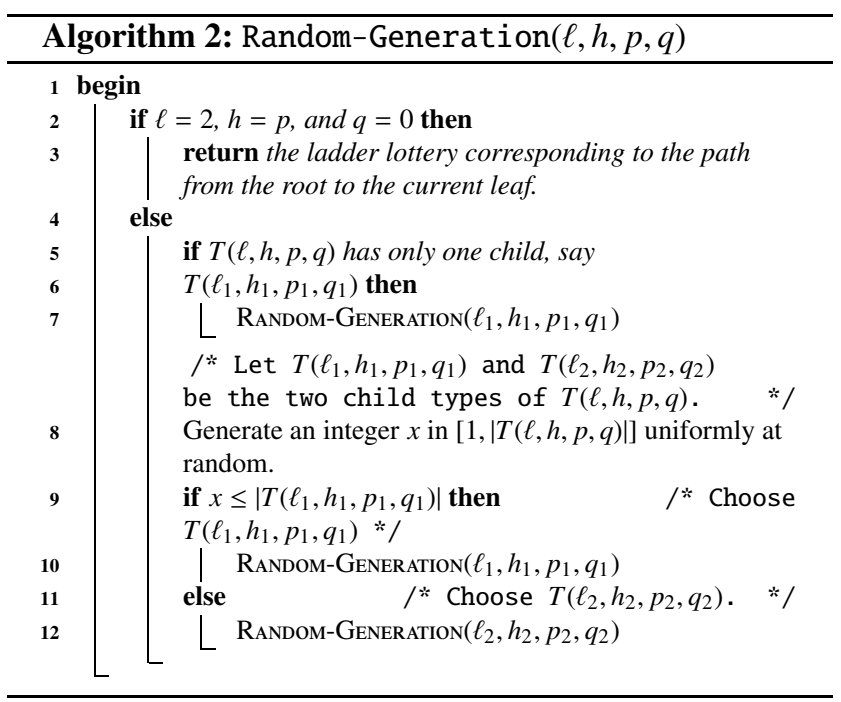

say $x$, in $[1,|T(\ell, h, p, q)|]$ uniformly at random, chooses $T\left(\ell_{1}, h_{1}, p_{1}, q_{1}\right)$ if $x \leq\left|T\left(\ell_{1}, h_{1}, p_{1}, q_{1}\right)\right|$ and $T\left(\ell_{2}, h_{2}, p_{2}, q_{2}\right)$ otherwise, then recursively call with the chosen type. Since Algorithm 1 computes the table as the preprocessing, these numbers can be looked up in $\mathrm{O}(1)$ time. Thus we can generate ladder lotteries uniformly at random, as in the following theorem.

Theorem 4: Given two integers $n \geq 2$ and $b \geq 0$, after computing the table of $|T(\ell, h, p, q)|$ by Algorithm 1, we can generate a ladder lottery with $n$ lines and $b$ bars in $\mathrm{O}(n+b)$ time for each, uniformly at random.

\section{Conclusion}

We have designed three algorithms for enumeration, counting, and random generation of ladder lotteries with $n$ lines and $b$ bars. All the three algorithms are based on the code [1] of ladder lotteries.

Our enumeration algorithm enumerates all the ladder lotteries with $n$ lines and $b$ bars in $\mathrm{O}(n+b)$ time for each. Our counting algorithm counts the number of ladder lotteries with $n$ lines and $b$ bars in $\mathrm{O}\left(n b^{3}\right)$ time. Our random generation algorithm takes $\mathrm{O}\left(n b^{3}\right)$ time as a preprocessing, then generates ladder lotteries with $n$ lines and $b$ bars in $\mathrm{O}(n+b)$ time for each, uniformly at random.

\section{Acknowledgments}

This work is partially supported by MEXT/JSPS KAKENHI, including the ELC project. (Grant Numbers 24106007 and 16K00002.)

\section{References}

[1] T. Aiuchi, K. Yamanaka, T. Hirayama, and Y. Nishitani, "Coding ladder lotteries," In Proceedings of European Workshop on Computational Geometry 2013, Braunschweig, Germany, pp.151-154, March 2013. 
[2] D. Avis and K. Fukuda, "Reverse search for enumeration," Discrete Applied Mathematics, vol.65, no.1-3, pp.21-46, 1996.

[3] J. Kawahara, T. Saitoh, R. Yoshinaka, and S. Minato, "Counting primitive sorting networks by $\pi$ DDs," Hokkaido University, Division of Computer Science, TCS Technical Reports, TCS-TR-A-11-54, 2011.

[4] D.E. Knuth, "Axioms and hulls," LNCS 606, Springer-Verlag, 1992.

[5] L. Manivel, Symmetric Functions, Schubert Polynomials and Degeneracy Loci, American Mathematical Soc., 2001.

[6] S. Nakano, "Efficient generation of triconnected plane triangulations," Computational Geometry: Theory and Applications, vol.27, no.2, pp.109-122, 2004

[7] N.J.A. Sloane, The on-line encyclopedia of integer sequences, Published electronically at https://oeis.org/A006245. Accessed: 2016-0724.

[8] K. Yamanaka and S. Nakano, "Listing all plane graphs," Journal of Graph Algorithms and Applications, vol.13, no.1, pp.5-18, 2009

[9] K. Yamanaka, S. Nakano, Y. Matsui, R. Uehara, and K. Nakada, "Efficient enumeration of all ladder lotteries and its application," Theoretical Computer Science, vol.411, no.16-18, pp.1714-1722, 2010.

\section{Appendix: Traversing Family Forest}

If we can traverse the family forest $F_{n, b}$, then we can enumerate all the ladder lotteries in $\mathcal{S}_{n, b}$, which correspond to the leaves of $F_{n, b}$. To traverse the family forest, we consider enumerating all root pre-ladders of $F_{n, b}$, and enumerating all child pre-ladders of a pre-ladder in $F_{n, b}$. First, by enumerating pre-ladder with two lines and $p$ unmatched l-bars attached to the first line for each $p=1,2, \ldots, b$, we obtain all root pre-ladder of $F_{n, b}$. We next consider to enumerate all child pre-ladders of any pre-ladder in $F_{n, b}$. Now imagine to generate the code corresponding to a child pre-ladder from the code corresponding to the parent pre-ladder. The code of a child pre-ladder is obtained by appending $\theta$ or 1 to the code corresponding to the parent pre-ladder as the second last bit. That is, a child pre-ladder is obtained by appending a half-bar or a line to the parent pre-ladder. Now we explain the details.

Let $R$ be a pre-ladder in $F_{n, b}$, and let $C(R)$ be the code of $R$. Recall that $R$ is a pre-ladder derived from some ladder lottery with $n$ lines and $b$ bars. We introduce a notation, as follows. $R(0)$ is the pre-ladder corresponding to the code obtained from $C(R)$ by appending $\theta$ as the second last bit. Similarly, $R(1)$ is the pre-ladder corresponding to the code obtained from $C(R)$ by appending 1 as the second last bit. Note that $R(0)$ is obtained from $R$ by attaching a r-bar or appending a line, and $R(1)$ is obtained from $R$ by attaching an l-bar. $R(0)$ and $R(1)$ are candidates of child pre-ladders of $R$. $R(i)$ for each $i=0,1$ is a child pre-ladder of $R$ if and only if $R(i)$ is a pre-ladder in $F_{n, b}$

We assume that $R$ has $\ell$ lines, $h$ half-bars, $p$ unmatched 1-bars attached to the $(\ell-1)$-th line, and $q$ unmatched 1-bars attached to the $\ell$-th line. Recall that a bar is regarded as a pair of an l-bar and an r-bar. If $R$ is a ladder lottery with $n$ lines and $b$ bars, then $R$ has no child. We hence assume that $R$ is not a ladder lottery with $n$ lines and $b$ bars. Note that $R$ is ladder lottery with $n$ lines and $b$ bars if and only if $R$ satisfies $\ell=n, h=2 b, p=0, q=0$.

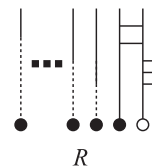

$R$

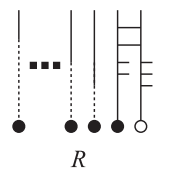

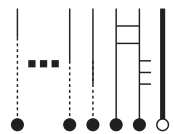

$R(0)$

(a)

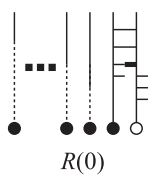

(b)

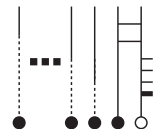

$R(1)$

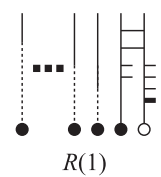

$R(1)$
Fig. A. 1 Illustration for child generations.

Case 1: $p=0$

We first assume that $\ell=n$ holds. Since there is no unmatched l-bar attached to the (n-1)-th line in $R, R(0)$ contains $n+1$ lines. (Note that, in this case, $R(0)$ is obtained from $R$ by appending a new line.) Hence, $R(0)$ is not a child pre-ladder of $R . R(1)$ includes an unmatched l-bar attached to the $n$-th line. Note that $R(1)$ is obtained from $R$ by appending an unmatched l-bar attached to the $n$-th line. Hence, $R(1)$ is not a child pre-ladder of $R$.

We next assume that $\ell=n-1$ holds. If $h+q<2 b$ holds, then we show that $R(0)$ is not a child pre-ladder of $R$ and $R(1)$ is a child pre-ladder, as follows. $R(0)$ contains $n$ lines and $q$ unmatched 1-bar such that $h+q<2 b$ holds. Hence any pre-ladder derived from $R(0)$ cannot contain $n$ lines and $2 b$ half-bars, namely, $b$ bars. Hence, $R(0)$ is not a child pre-ladder. On the other hand, $R(1)$ is a child preladder of $R$. If $h+q=2 b$ holds, then we show that $R(0)$ is a child and $R(1)$ is not a child. $R(0)$ is obtained from $R$ by appending a new line. Hence, $R(0)$ is a child pre-ladder of $R$. Since $h+q=2 b$ holds, any unmatched l-bar cannot be appended to derive a ladder lottery with $n$ lines and $b$ bars. Hence, $R(1)$ is not a child pre-ladder.

We finally assume that $\ell<n-1$ holds. If $h+q<$ $2 b$ holds, then both $R(0)$ and $R(1)$ are child pre-ladders, as illustrated in Fig. A- 1 (a). If $h+q=2 b$ holds, then only $R(0)$ is a child pre-ladder.

Case 2: $p>0$

In this case, we can assume that $h<2 b$ holds. (If $h=$ $2 b$ holds, we cannot append any r-bar such that it matches to an l-bar attached to the ( $\ell-1)$-th line.) If $h+p+q<2 b$ holds, then both $R(0)$ and $R(1)$, as illustrated in Fig. A. 1 (b), are child pre-ladders.

Next, we assume that $h+p+q=2 b$ holds. Then $R(0)$ is a child pre-ladder. On the other hand, $R(1)$ is not a child pre-ladder, since any pre-ladder in $\mathcal{S}_{n, b}$ cannot be derived from $R(1)$.

By the case analysis above, we have the algorithms shown in Algorithm 3 and Algorithm $4 . \quad$ Algorithm 3 is the main routine. It enumerates all root pre-ladders of $F_{n, b}$, then calls Algorithm 4 (FIND-ALL-ChILDREN) for each root pre-ladder. Algorithm 4 recursively enumerates all child pre-ladders of a given pre-ladder, so it traverses the 

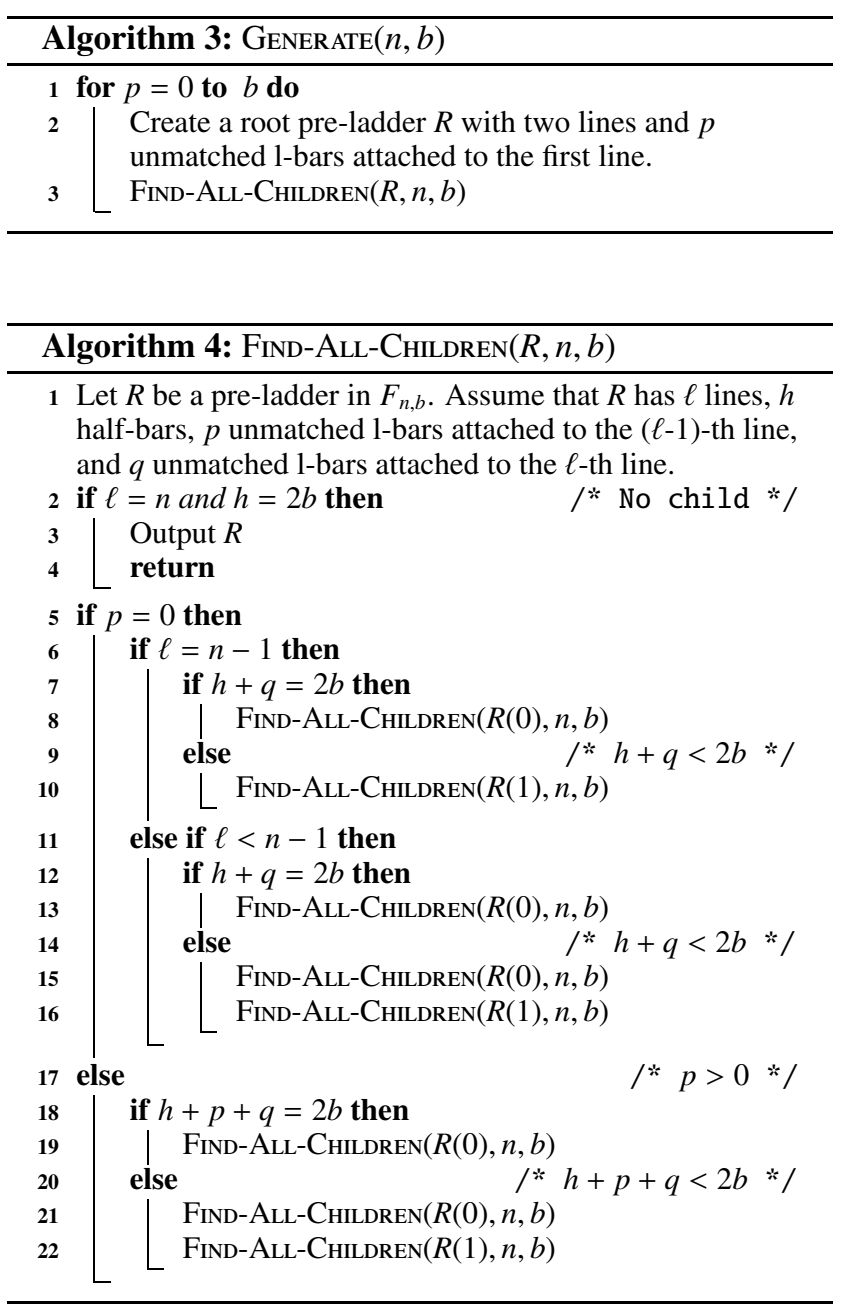

family forest and output a ladder lottery at each leaf. Algorithm 4 always stores the current pre-ladder in global memory and updates it with some difference information which is needed to reconstruct the previous pre-ladder. Hence, memory space required in our algorithm is $\mathrm{O}(n+b)$. By Algorithms 3 and 4 , each child pre-ladder can be generated in $\mathrm{O}(1)$ time. We have the following theorem.

Theorem 5: Our algorithm uses $\mathrm{O}(n+b)$ space and enumerates every ladder lottery with $n$ lines and $b$ bars in $\mathrm{O}(n+b)$ time for each.

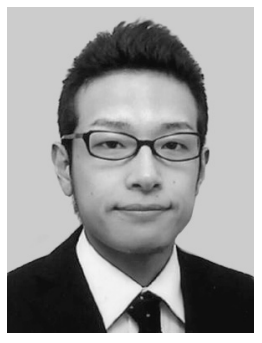

Katsuhisa Yamanaka is an assistant professor of Department of Electrical Engineering and Computer Science, Faculty of Engineering, Iwate University. He received B.E., M.E. and Dr. Eng. degrees from Gunma University in 2003, 2005 and 2007, respectively. His research interests include combinatorial algorithms and graph algorithms.

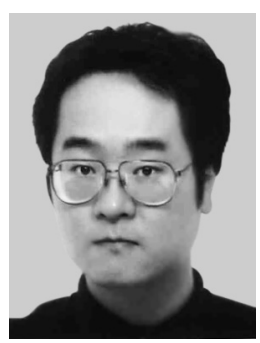

puter Society.
Shin-ichi Nakano received his B.E. and M.E. degrees from Tohoku University, Sendai, Japan, in 1985 and 1987, respectively. In 1987 he joined Seiko Epson Corp. and in 1990 he joined Tohoku University. In 1992, he received Dr. Eng. degree from Tohoku University. Since 1999 he has been a faculty member of Department of Computer Science, Faculty of Engineering, Gunma University. His research interests are graph algorithms and graph theory. $\mathrm{He}$ is a member of IPSJ, JSIAM, ACM, and IEEE Com- 